\title{
Post-COVID-19 return to elective orthopaedic surgery-is rescheduling just a reboot process? Which timing for tests? Is chest CT scan still useful? Safety of the first hundred elective cases? How to explain the "new normality health organization" to patients?
}

\author{
Jacques Hernigou ${ }^{1}$ - Jérome Valcarenghi ${ }^{2}$. Adonis Safar ${ }^{1}$ - Mohamed Amine Ferchichi ${ }^{1}$ - Esfandiar Chahidi ${ }^{2}$. \\ Harold Jennart $^{2} \cdot$ Philippe Hernigou $^{3,4}$ (D)
}

Received: 24 June 2020 / Accepted: 10 July 2020 / Published online: 19 July 2020

(C) SICOT aisbl 2020

\begin{abstract}
Purpose The long incubation period and asymptomatic spread of COVID-19 present considerable challenges for health care institutions when patients return to elective surgery.

Methods A retrospective review of the first adult elective cases performed between May 18, 2020 and June 14, 2020, after the end of lockdown was analysed in Belgium to answer the following questions: (1) for the 236 cancelled patients during the outbreak, how easy was rescheduling? (2) How useful was universal RT-PCR testing and chest CT scan for the 211 orthopaedic and trauma admissions? (3) How were surgical difficulty category, number of operations and complications different when compared to the pre-COVID period? (4) How would patients balance the benefit of surgery against the unknown risk of developing COVID-19?

Results Before surgery, blood tests for anaesthesiology and imaging related to the surgical procedure were scheduled prior to universal testing (COVID-19 PCR and chest CT) performed 72-120 hours before surgery. Among the 211 asymptomatic patients who were tested before surgery, six had positive PCR, while no abnormality was found on the chest CT scan of all the patients. With this timing for tests, the 104 patients included in the current study for elective surgery were free of disease before undergoing surgery and remained without COVID-19 after surgery. Among the 366 cancelled patients during the outbreak, only $12 \%$ of the patients accepted to proceed with rescheduling immediately. Therefore, this resulted in a $70 \%$ reduction for elective surgery and in a 50\% reduction for arthroplasties as compared to pre-COVID period. The rate of complications was not increased during the post-COVID period. A portion of patients have confused idea of screening and have difficulty to perceive the new rules of health organization.

Conclusions Resumption of elective surgical procedures appears more difficult for patients than for surgeons with a low percentage of cancelled patients accepting to reschedule surgery. Universal testing allowed securing patients; however, surgeons must explore better patient perceptions regarding COVID-19 to facilitate a fully informed decision in the current period.
\end{abstract}

Keywords Post-COVID-19 · Elective surgery · Hip arthroplasty $\cdot$ Fracture $\cdot$ Surgery cancellation · Ethics · Chest CT scan · Psychological burden

Philippe Hernigou

philippe.hernigou@wanadoo.fr

1 Orthopedic Department, EpiCURA Baudour Hornu Hospital, Mons, Belgium

2 Orthopedic Department, Tivoli hospital, La Louvière, Belgium

3 Orthopedic Department, Clinique Geoffroy Saint Hilaire, 75005 Paris, France

4 Hospital Henri Mondor, University Paris, Paris, France
With 24,809 articles on PubMed for "COVID-19" at the end of June 2020, there are many hypotheses and theories [1-5] about the best road to recovery after lockdown; but to our knowledge, there are no data (even from China) on the "post-COVID-19" clinical characteristics and outcomes of patients who had had elective orthopaedic surgery. In many countries of Europe, and in many other countries, governments provided guidance recommending or mandating the discontinuation of elective procedures. There were several reasons for this recommendation, among them: preserve 
critically short supplies of respirators and preserve inpatient bed capacity and other equipment for critically ill patients. The short-term benefits are clear: the risks of exposure to COVID-19 are reduced, and the associated complications have been decreased. The consequences (after end of lockdown) are at this moment unknown.

As the end of lockdown arrived in Belgium, the authorization to perform elective surgery again was obtained in 18 May 2020 and orthopaedic institutions across the country have begun to resume elective orthopaedic cases. Moving towards this direction required changes to protect hospital staff and to reassure patients who expect a low risk of exposure for these elective surgical procedures. As such, virus-free perioperative pathways should be established at each institution. Unfortunately, the ability of the virus to spread from asymptomatic patients and the prolonged incubation time make this proposition difficult. Furthermore, during the same period, trauma with potential infected patients can continue to arrive in the institutions.

In this study, we aim to present the epidemiological, clinical characteristics, treatment and outcomes of patients undergoing elective surgery after the end of lockdown. Particularly, the aim of this study was to report the following points: (1) among patients who had their elective surgery cancelled, how easy was rescheduling? (2) An institutional policy occurred on May 18, 2020, which indicated nasopharyngeal swab RT-PCR testing and chest CT scan for all orthopaedic and trauma admissions among $\mathrm{X}$ patients. How useful were screening, timing, and testing data for COVID-19 as well as relevant imaging? (3) How were surgical difficulty category, number of operations performed and number of complications different when compared to the pre-COVID period? (4) How was the perception of patients when balancing the benefit of surgery against the unknown risk of developing COVID19 ?

\section{Material and methods}

\section{Study design}

This study is a retrospective multi-centric review of all elective surgeries and trauma surgery that were performed in Belgium at two institutions (EpiCURA-Baudour/Hornu and Tivoli-La Louvière hospitals) between May 18, 2020 and June 14, 2020. The start date was chosen as May 18, 2020 as this was the date that our institution after the end of lockdown converted to performing elective orthopaedic cases. The number of elective operation performed on this month basis was analysed, as the number of new COVID-19 cases admitted to our institution and the prevalence of COVID-19 within the Belgium population at the same moment.
Surgery was performed with "hospital policy" concordance: without jeopardizing the stock of essential items, while maintaining safety of personnel and patients. We analysed the impact of cancelling elective surgery on the hospital activity; the efficiency to test everybody (universal testing) before surgery; the difficulty, number and complications of operations and the epidemic perception by patients.

\section{Rescheduling cancelled patients}

Postponed cases were rescheduled considering the patient's desire to proceed with elective surgery, the clinical urgency of the treatment, the resources required to do the case (staff, number of hospital days, etc.) and the delay that the patient had experienced due to the COVID-19 postponement.

Usually, cancellation of surgery results from an agreement between patient, surgeon and anaesthesiologist due to medical considerations and/or patient-related reasons (unrelated illness, medically unfit or surgery declined). COVID-19 cancellation was a government decision; therefore, the effects of cancellation may be different from those observed when it is a patient decision. We analysed this effect on the rate of patients who rescheduled their operations, on the economic loss for patients and on the psychological burden.

When patients decline to reschedule their operations, we analysed two essential causes:

- Financial cost for working people: the economic impact of a cancellation on patients themselves has received remarkably little attention. Most patients in their preparation for elective surgery make arrangements in their working lives to facilitate this. Adults in employment have to book time off work and sometimes postpone events such as holidays. If a surgery is cancelled at short notice, this may result in an economic loss, and rescheduling can cause additional loss of working days with economic impact.

- Psychological impact: cancellation of elective surgery may also cause anxiety on patients who have "mentally" prepared this event. This anxiety may be associated with a longer wait for surgery, with fear that their condition deteriorates further. Another issue is balancing the benefit of surgery against the unknown risk of developing COVID-19 and its associated complications when going to surgery in hospital.

Patients were asked to answer to a questionnaire when they decline to reschedule their operation, choosing financial reason or psychological impact. They were allowed to provide a free "short text response" (one or two words) for the cause of cancellation. 


\section{Tests (PCR and chest CT) and timing of testing for COVID-19}

As explained in Fig. 1, during the apex of the pandemic, the number of new cases per day was high and decreased quickly after the 18th if May 2020.

Before end of lockdown (18 May 2020), screening of all patients for COVID-19 was instituted as previously reported [6], with a questionnaire and temperature testing. The questionnaire queried about onset of symptoms, fever of $38^{\circ} \mathrm{C}$, cough, shortness of breath, muscle aches, respiratory difficulty, pneumonia, nausea, headache, abdominal pain and loss of taste or smell. Patients scheduled for admission who screened positive were tested with a nasopharyngeal swab RT-PCR for COVID-19, and some (but not all) had chest CT scan. Patients who screened negative on questionnaire were sometimes not tested.

After end of lockdown (18 May 2020), our institutional policy for pre-operative COVID-19 testing was changed, to include pre-operative testing of any patient (symptomatic or asymptomatic) with the chance for post-operative admission. Patients having elective surgery were tested at one of the outpatient swab sites with a polymerase chain reaction (PCR)-based protocol because in most instances, the rapid antibody test has a lower accuracy especially among asymptomatic patients. These patients had also for the first four weeks after lockdown a chest CT scan [7] as previously reported during lockdown. Timing of the COVID-19 swabbing test depends on the patient's day of surgery. The patient needs to obtain the test $72-120$ hours prior to operation to ensure adequate time for result prior to operation. After the test, the patient should remain at home with minimum contact with other people between COVID19 testing day and planned surgery day. Ancillary testing (e.g. blood work, imaging) related to the surgery procedure needed to be scheduled prior to COVID-19 testing.

\section{Surgical difficulty category, number of operations performed and complications}

A comparison between a prospective cohort study of all patients operated after returning to elective surgery between 18 May 2020 and 14 June 2020 and a retrospective control group of patients operated in May-June 2018 was undertaken. Data on each patient admitted to our hospital with an admission for elective surgery or trauma were collected. These included demographics, diagnosis, type of anaesthesiology and surgery and time interval between admission and surgery.

Anaesthesiology was not different and treatments were similar. Indications and treatments were similar. Prophylaxis with low-molecular-weight heparin (LMWH), standard hydration, was administered according to our hospital's protocol for patients who needed in a similar way during the two periods.

The patients included in this study underwent various surgical procedures and were categorized into four levels based on the degree of technical difficulty, complexity and risk:

Briefly, level-1, various operations with low risks, simple procedures and low technical difficulty as material removal; Level-2, various operations with mild risks, general complexity of procedures and general technical difficulty in young patients as arthroscopy, osteotomy, tendon and ligament repairs;Level-3, various operations with moderate risks, complex procedures and moderate technical difficulty in patients older than 65 years as hip and knee arthroplasty; shoulder arthroplasty and Level- 4, various operations with high risks, highly complex procedures and high technical difficulty as revision arthroplasty, spine arthrodesis.

Complications as bacteriological infections, thrombophlebitis, and pulmonary embolisms were evaluated in the two periods.

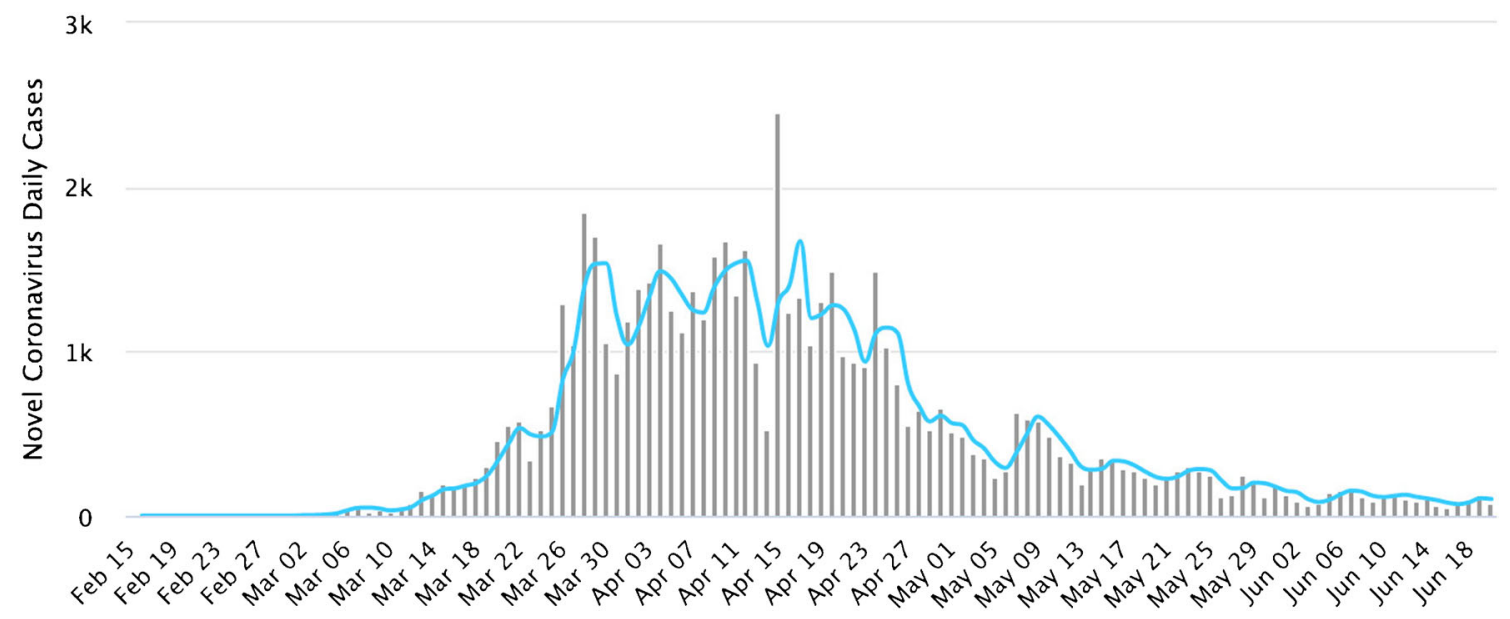

Fig. 1 Daily new cases from 1 March to 14 June 2020; $1 \mathrm{k}=1000$ cases 


\section{Information for patients on "new" health situation}

Despite the benefits of early identification and expanded screening criteria, multiple diagnostic barriers currently exist. One such barrier is that many patients falsely believe that they do not require screening or at the reverse that they were tested during a prior hospital visit. The magnitude of this effect has not been clearly defined in the literature. Another critical issue is balancing the benefit of surgery against the unknown risk of developing COVID-19 and its associated complications. As the magnitude of this effect has not been defined in the literature, we evaluated this problem with a questionnaire among patients.

These questions included beliefs regarding specific tests performed at current admission, and whether the patient had experienced a prior hospital admission or medical visit within the last four weeks. If the patient had a prior hospital or medical visit, the patient was asked questions regarding whether they believed that they received COVID-19 testing at that time, and if so, the patient was asked if they believed that not receiving a test result at that time was the equivalent of receiving a negative test result. All the patients were also asked if they had ever been offered a screening test and whether they had knowledge of the disease.

Another issue was exploring patient perception of the disease by asking the patient if he (she) would have refused to undergo urgent surgery and rather chosen plaster treatment when available (even with lower result expectation) due to the fear of contracting COVID-19 when the governmental decision has determined that the viral risk to the population was greater than the risk of cancelling all routine procedures. Patients were also asked about their feeling after the end of lockdown concerning how clean the hospital is concerning eliminating COVID-19 risk: totally or partially?

\section{Statistical analysis}

Continuous variables were presented as mean with ranges and compared by using independent-group $t$ tests when the data were normally distributed; otherwise, the Mann Whitney test was used. Categorical variables were expressed as frequencies and percentages and compared by chi-square or Fisher's exact test between groups.

\section{Results}

\section{Cancellation of 366 operations: only 44 patients accepted to reschedule operations}

Of the 366 patients who had elective surgery cancelled, only $44(12 \%)$ accepted to get surgery rescheduled during the first month after lockdown. Of these 366 patients with a cancelled surgery, $40(11 \%)$ had their operations cancelled on the week of surgery, $90(25 \%)$ within two weeks of the proposed date of surgery and the others were cancelled more than three weeks from the proposed date of the operation. Despite a high percentage of cancellation on the week of surgery, we were unable to demonstrate any correlation between the period for cancellation of the planned operation, and the impact on the fact that patients refuse to reschedule their operation.

\section{Four (1\%) patients could not reschedule their operation since they were hospitalized for COVD-19.}

Among the 44 patients who accepted to reschedule operation, $5(12 \%)$ had had COVID-19. Among the other 322 patients, $16(5 \%)$ had had COVID-19. A higher number of patients who had disease were found among patients who accepted to reschedule surgery, these patients feeling probably that they were no more concerned about the risk of entering a health care facility.

Economic burden was the principal cause of refusing to reschedule a new operation: following the lockdown period, when surgery was rescheduled, $72 \%$ (232 among the 322) of patients could not have surgery performed due to the economic problem; patients had to work and could not afford any time to reschedule lower limb procedures.

Psychological burden: $18 \%$ (60 among 322) of the patients reported words as sadness, disappointment, anger, frustration and stress; they were reluctant to undergo procedures while the pandemic simmers and their risk of infection was considered as high by themselves. The qualitative analysis of the participants' free text responses indicates that there was also concern over symptoms worsening. However, the most interesting finding was that $90 \%$ of the responders understood the reason for cancellation of their operations in order to facilitate delivery of emergency service. They used such descriptions as: "my surgery was not life-threatening" and "others people needed the hospital more".

\section{Tests and timing of testing for COVID-19 among the 211 patients with surgery}

Testing results (PCR and chest CT scan) under the universal protocol for elective surgery

In this study, the 104 adult patients scheduled for elective orthopaedic surgical procedure were screened for COVID19 with PCR, and 88 patients also were screened with chest CT scan (Table 1). No CT scan was positive for COVD-19. Among the four weeks following the lockdown period, in total, five patients were tested pre-operatively positive for COVID-19. These five patients were asymptomatic with a 
Table 1 Comparison of PCR and chest CT scans results (number of patients) between lockdown and post-lockdown periods

\begin{tabular}{lll}
\hline & Lockdown period & Post-lockdown period \\
\hline & Patient's number & Patient's number \\
PCR & 47 & $211(104+107)$ \\
Positive & $15(34 \%)$ & $5(2.3 \%)$ \\
Negative & $32(66 \%)$ & $206(97.7 \%)$ \\
Chest CT scans & 118 & $180(88+92)$ \\
Positive & $16(14 \%)$ & 0 \\
Negative & $102(86 \%)$ & $180(100 \%)$ \\
\hline
\end{tabular}

negative CT scan. Finally, when considering the screening value of both PCR and chest CT scan findings, the CT scan was of no value in this post-lockdown period contrary to the value observed during the pandemic apex period [7].

\section{Tests and chest CT screening for trauma patients}

Meanwhile, 107 patients had trauma surgery; these 107 were screened for COVID-19 with PCR, and 92 patients also were screened with chest CT scan (Table 1). None was symptomatic at the time of diagnosis. In total, only one patient was tested positive for COVID-19, but the result was obtained after surgery (normal CT scan). As compared with the pandemic apex period, there was a huge decrease of frequency of positive tests $[6,7]$.

\section{Indirect information from normal chest CT scan for surgery}

During the apex of the pandemic, in the same geographic area, $15 \%$ of patients had pneumonia on chest CT scan [7] while being asymptomatic. We can suspect that about $30(15 \%)$ of our 211 asymptomatic patients had during the apex of the pandemic an abnormal chest CT scan. As all chest CT scans were normal at the post-COVID period, the chest CT scan appears to return to normality in asymptomatic patients; they have probably no sequels of an eventual pneumonia and the chest CT scan is of no value to detect sequels in asymptomatic patients. At the contrary, patients with a previous symptomatic disease may have pneumonia sequels and may need chest $\mathrm{CT}$ for anaesthesiologist evaluation.

\section{Surgical activity: decrease elective operations performed, but no complications}

\section{Elective surgery}

During the months of May-June 2018 selected as a baseline reference, the volume of arthroplasties and other elective surgeries performed in our departments was 705 elective operations, which translates to an average weekly volume of 79 elective procedures, and among these operations, 12 arthroplasties (hip, knee, shoulder) or revision arthroplasties were performed weekly.

During the COVID-19 lockdown pandemic period (7 weeks), 12 non-deferrable elective surgical procedures were performed; no arthroplasty was performed as elective surgery.

After returning to elective surgery, 104 elective surgeries (25 arthroplasties) were performed during the first four weeks (Tables 2 and 3); 44 of these 104 patients (mean age 54 years; range 21 to 82 years) were patients previously cancelled (18 arthroplasties). Therefore, there was a $70 \%$ reduction for elective surgery and a 50\% reduction weekly for arthroplasties. A review of medical records and readmissions showed that none of the orthopaedic patients in our institution were diagnosed with COVID-19 infection before or after surgery.

In the present study, 102 patients underwent operations with the surgical difficulty category at level-1 or level-2 and level-3; only two patients underwent revision arthroplasties with the surgical difficulty category at level-4, the highest surgical difficulty category. No patients required additional oxygen supplementation post-operatively. No patients were admitted to intensive care unit (ICU).

During the baseline reference of 2018, there was a higher number of level-4 surgery (Table 3). Limiting the comparison to level-1, level-2 and level-3 surgery, the number of complications (no bacteriological infections, no symptomatic thrombophlebitis, and no pulmonary embolisms in 2020) was not higher during the post-COVID pandemic.

Table 2 Elective procedures during same periods in 2018 and 2020

\begin{tabular}{lll}
\hline & $\begin{array}{l}\text { 18 May-14 June 2020 } \\
\text { Elective operations }\end{array}$ & $\begin{array}{l}\text { 1 May-30 June 2018 } \\
\text { Elective operations }\end{array}$ \\
\hline Nb of surgeries & 104 & 705 \\
Nb of weeks & 4 & 9 \\
Operations/week & 26 & 79 \\
Sex & & \\
Man & $56(54 \%)$ & $367(52 \%)$ \\
Woman & $48(46 \%)$ & $338(48 \%)$ \\
Age & $54.5(\mathrm{CI} 95$ 51.4-57.5) & $57.4(\mathrm{CI} 9555.8-58.9)$ \\
Arthroplasties/week & 6 & 12 \\
Arthroplasties & 25 & 112 \\
Hips & 11 & 52 \\
Knees & 11 & 41 \\
Shoulders & 1 & 11 \\
Revision TKA & 1 & - \\
Revision THA & 1 & 8 \\
\hline
\end{tabular}

$T K A$ total knee arthroplasty, THA total hip arthroplasty 
Table 3 Population description of elective and trauma surgeries groups in the post-COVID-19 period

\begin{tabular}{|c|c|c|}
\hline & Elective surgery & Trauma surgery \\
\hline Number of patients & 104 & 107 \\
\hline \multicolumn{3}{|l|}{ Sex } \\
\hline Man & $56(54 \%)$ & $48(45 \%)$ \\
\hline Woman & $48(46 \%)$ & $59(55 \%)$ \\
\hline Age & 54.5 (CI95 51.4-57.5) & 56.6 (CI95 52.3-61) \\
\hline Arthroplasties & 25 & 11 \\
\hline Hips & 11 & 11 \\
\hline Knees & 11 & - \\
\hline Shoulders & 1 & - \\
\hline Revision TKA & 1 & - \\
\hline Revision THA & 1 & - \\
\hline
\end{tabular}

TKA total knee arthroplasty, THA total hip arthroplasty

\section{Trauma surgery}

During the months of May-June 2018 selected as a baseline reference, 175 admissions for adult fracture surgery were observed, which translates to an average weekly volume of 19 trauma procedures.

During the COVID-19 lockdown pandemic period (7 weeks), the number of trauma patients during the pandemic 2020 was significantly lower for adults (80 cases), corresponding to 11 trauma procedures each week.

After lockdown, 107 trauma patients (mean age 57 years; 18 to 98 ) were treated (Table 3), returning to 27 procedures weekly. Only one asymptomatic patient was positive for PCR COVID-19; he needed additional oxygen supplementation post-operatively without requiring intubation and ICU admission. Twenty-six patients with an average age of 77 years (range 39 to 98 years) were admitted to the emergency orthopaedic room for the treatment of a proximal femoral fracture; 14 femoral neck fractures were treated with arthroplasty and none needed ICU care.

\section{Information for patients on health situation and pertinent changes}

One hundred and sixteen patients completed the survey. Sixty (52\%) patients believed that they had received a screening test for COVD-19 during a previous medical visit. Patients with elective surgery were more likely to believe that they had received at least one screening test (61\% vs. $39 \%)$ as compared to trauma patients. Patients believed that it was a serologic test during the anaesthesiologist visit. Others who were tested PCR negative one time were thinking that they would be indefinitely negative.
Among the 60 patients who believed that they had a prior hospital-associated screening test, $82 \%$ interpreted the lack of result as equivalent to a negative test result. Overall, among those patients with a prior hospital visit, this results into approximately $25 \%$ of patients who believe that they were screened for COVID, and for them, absence of results was equivalent to negative for disease.

For patient perception risk of the disease, 18 (15.5\%) patients would have refused to undergo urgent surgery during the pandemic and would have chosen plaster treatment (when available even if the result could have been inferior) due to the fear of contracting COVID-19 during the pandemic. When patients were asked how clean the hospital is after lockdown, concerning eliminating COVID-19 risk, 37 (32\%) answered totally and $68 \%$ partially.

\section{Discussion}

Regardless of definition, cases should be resumed in a graduated fashion as the outbreak subsides, as COVID-19 will be persistent and "hot-spot outbreaks" or local surges are still possible. The availability of appropriate numbers of intensive care unit (ICU) and non-ICU beds at a given hospital, the availability of personal protective equipment (PPE) and ventilators and the available compliment of trained staff to perform non-essential surgical procedures without taxing resources need to deal with the ongoing COVID-19 crisis.

\section{High impact of cancellation on patient's difficulties and on orthopaedic activity}

In many countries, governments provided guidance mandating the discontinuation of elective procedures. The consequence in Belgium was a storm of surgical cancellations during the spring of 2020 from 15 March to 18 May 2020. The national strategy, instigated by the Belgium government, was to cancel elective operations to release capacity in the system. The pandemic and elective cancellations were publicized in the media and meant that the majority of the population had some idea of the unusual stress the health system was under. However, despite public awareness, the impact on patients of these cancellations was significant.

Cancellation surgery is not a new phenomenon but usually is limited and results from an agreement between patient and surgeon and is due to medical considerations. Usually, when cancellation is related to medical consideration, $90 \%$ of the patients accept to reschedule immediately [8] their surgery. This study is the first to evaluate the impact of the mass cancellation (related to the pandemic) of elective surgery on patients, from both an economic and psychological view point. Taken in the context of a nation-wide bed pressure crisis, which was widely publicized in the media, the fact that nearly 
$88 \%$ of patients did not accept rescheduling of their surgery may be surprising.

Many patients could not reschedule surgery due to the negative economic impact. Following the COVID-19 crisis, patients had economic, employment and insurance concerns; the demand for surgery was lower than expected. Younger patients were not able to delay work for long periods of time and when the lockdown was finished, they had to work and could not schedule another date for surgery. Therefore, protocol for patient preference for timing of rescheduling their operation should be established. For example, some patients may not want to pursue rescheduling their operation at the moment and may not want to initiate further discussion about rescheduling in the future. Alternatively, other patients may be unsure about rescheduling their operation at the current time but would likely reschedule at a later date; other patients may want to proceed with rescheduling immediately.

Very little information is available on the psychosocial impact of cancellations of elective operations on patients after COVID-19. The questions on psychological impact have demonstrated that elective surgical cancellation led to negative emotion among patients, with most of them displaying some health-related anxiety. Although this study was about spring cancellations of operations of patients from one region of Europe, the results are probably generalizable to other countries and other dates.

\section{Universal testing (PCR still useful; chest CT of no more value)}

Due to risk of some asymptomatic patients being COVID-19 positive, asymptomatic patients undergoing an elective procedure with "risk for aerosol generation" should undergo COVID-19 testing prior to a surgical procedure. Using routine nasopharyngeal RT-PCR testing and chest CT scan for planned essential surgical admissions, our study shows a COVID-19 prevalence of 2.8\% (6 among 211 patients) in Belgium during the four week period following the apex of the pandemic. This lies in stark contrast to the rate detected during the preceding four week period throughout which testing was not routine.

\section{Chest CT scan is no longer useful outside the pandemic period}

During the apex of the pandemic, among 254 patients, $15 \%$ (38 patients) had a positive COVID-pattern CT scan. During this period, among the 118 patients with chest $\mathrm{CT}$, there were $16(13.5 \%)$ patients with positive chest CT findings, and useful information for the orthopaedic surgeon was obtained for $87(74 \%)$ patients of these 118 patients. During the apex of the pandemic, patients who had no symptoms, a negative RTPCR result and chest CT without evidence of interstitial pneumonia could be triaged to surgery at a COVID-19-free pathway. There was evidence that CT evaluation [7] was the most accurate diagnostic test for COVID-19 pneumonia in patients who needed surgery in emergency, but its use as a screening tool remains unnecessary for elective surgery since surgeon and patients can wait for the result of PCR during some days. Furthermore, patients with abnormalities on CT scan are no longer found, and interestingly, in asymptomatic patients who had eventual pneumonia, nosequels were found. So, except in patients who had a previous symptomatic infection with recovery and may have pulmonary sequels [9], CT scan is nomore useful. So, except in symptomatic patients who may have pulmonary sequels [9], CT scan is no longer useful.

\section{Nasopharyngeal testing with PCR is useful but with a limited efficiency}

In reporting these results, we hope to inform other institutions of the difficulty of screening patients for COVID-19 with PCR nasopharyngeal testing. Using universal testing even for asymptomatic patients, $2.8 \%$ patients were found positive. In recommending routine testing for pre-operative patients, we acknowledge the high specificity but only moderate sensitivity of the RT-PCR test as previously reported. But few other practical options exist. A new area of pre-operative testing involving antibody serology is possible, but studies have shown that people with the COVID-19 disease do not start antibody production until 11 or 12 days after symptom's onset. Thus, this test as a screening method in the early stages would be ineffective when negative. However, when positive, patients may feel more comfortable for an elective surgery with the knowledge that they may be immune to the disease when they have antibodies to the virus.

\section{Surgical difficulty category, number of operations performed and risks of complications}

This report is one the first retrospective studies describing the characteristics and outcomes of patients returning for elective surgery after the pandemic. None of the operated patients had sign or symptom of COVID-19 before surgery, and testing was able to secure these patients for elective surgery. The length of time between testing and surgery was shorter than the average incubation time ( 5 days) described in the literature. This supports our belief that the 104 patients with elective surgery were free of COVID-19 disease before operation and remained without disease after surgery.

Another concern involves the risk of undiagnosed COVID19-associated complications in the peri-operative period. As a silent window exists between contamination and positivity of the PCR, or seroconversion, a misdiagnosis of COVID-19 is always possible. The effects of surgical stress and medications 
on an eventual exacerbation of an asymptomatic disease are unknown. COVID-19 is associated with an abnormal thrombotic state [10] which might be a concern for orthopaedic patients who have risks of thromboembolic events. Elective surgery was performed as usual before the pandemic with the same preparation, the same preventive anticoagulation and the same antibiotics for prophylaxis. Our study illustrates that arthroplasties can be done when the disease curve decreases, provided that the resources and manpower remain available. We did not observe a higher rate of complications than before the pandemic for this population, and none of these patients received ICU care. Despite that we were able to treat only a small number of patients as compared to pre-COVID period, and despite that we performed instead level- 2 and level- 3 surgery, we hope that our findings will benefit the global community when returning to elective surgery.

The data on the impact of trauma surgery performed after the pandemic also are lacking. In our institution, the number of operations performed for trauma increased after the end of lockdown (May 18, 2020). Some of these patients were older, had more underlying comorbidities and longer surgical time and undergone more difficult surgery than patients with elective surgery. We did not observe complications.

Despite universal testing with chest CT scan and PCR, the probability of an asymptomatic and undiagnosed COVID-19 patient operated during this period exists and probably occured due to the frequency of the disease; data in this study suggest that surgery may not exacerbate disease progression of asymptomatic COVID-19, when the disease is present and undiagnosed at the time of surgery.

\section{Information for patients on health situation and pertinent changes}

Identification of COVID-19-positive patients is an important health issue, but successful screening faces many barriers. Our study clearly demonstrates that one such barrier is erroneous patient beliefs regarding prior screening tests during a previous medical visit. Approximately half of patients believed that they had received screening for COVID-19 despite never receiving information. The most concerning result was that the vast majority of these patients believed that the failure to receive a test result was equivalent to receiving a negative test result. These falsebeliefs may result in risk behaviours, with patients refusing testing that they would feel redundant or incorrectly reporting their previous screening to medical doctors.

Having a "clean" hospital with elimination of COVID19 risk was one demand on the questionnaire. However, despite the end of lockdown, persistent viral spread, asymptomatic cases and a silent window between contamination and a positive PCG or a seroconversion exist. Therefore, it would be very demanding as for all the epidemics [11] to keep a hospital clean (without any virus) for a long period of time and may be impossible even in the most developed countries with the most sophisticated techniques of decontamination. So, the question in elective orthopaedic surgery is: how can we ethically provide the traditional gold standard operation and explain the risks to patients during the COVID-19 crisis?

We have implemented an updated informed consent process that includes an explicit discussion about the steps the institution has taken to mitigate the risk of contracting COIVD-19 during the elective surgery, including preoperative testing, daily screening of staff and use of appropriate PPE. Patients should also be informed about visitor restrictions [12-14]. Our institution as many others [15-17] has made adjustments: a "no visitor" policy which extends to elective surgery. For major inpatient surgery, one person is allowed to accompany a patient on the day of surgery and one the day after surgery. During surgery, family and visitors are required to wait off site so as to avoid congregation and to encourage social distancing. Additionally, the surgeon should discuss that contracting COVID-19 could affect the postoperative recovery process and that rehabilitation services and post-operative care may be provided utilizing virtual visits [18] or telephone visits in order to limit in-person interactions.

\section{Conclusion}

As surgeons move forward to recovery of elective surgery, because undiagnosed asymptomatic COVID-19 patients remain a possibility $[19,20]$, surgeons must be aware that there are compromises between providing surgical care to patients and risk of spreading the virus. The COVID-19 crisis has placed a new type of discussion on the health care system in the world. Resumption of elective surgical procedures appears more difficult for patients than for surgeons with a low percentage of cancelled patients accepting rescheduling of surgery. Universal testing allowed safely securing patients; however, surgeons must explore patient's anxiety and perceptions regarding COVID-19 better to facilitate a fully informed decisions in the current period.

\section{Compliance with ethical standards}

Conflict of interest The authors declare that they have no conflict of interest.

Ethical approval All procedures were in accordance with the ethical standards of the institutional and/or national research committee and with the 1964 Helsinki declaration and its later amendments or comparable ethical standards.

Informed consent Informed consent was obtained from all individual participants included in the study. 


\section{References}

1. Hing CB, Al-Dadah O (2020) Returning to elective surgery, the 'new normal'. Knee 27(3):A1. https://doi.org/10.1016/j.knee.2020. 06.001

2. Zeegen EN, Yates AJ, Jevsevar DS (2020) After the COVID-19 pandemic: returning to normalcy or returning to a new normal? J Arthroplasty S0883-5403(20):30427-30427. https://doi.org/10. 1016/j.arth.2020.04.040

3. de Caro F, Hirschmann TM, Verdonk P (2020) Returning to orthopaedic business as usual after COVID-19: strategies and options. Knee Surg Sports Traumatol Arthrosc 28(6):1699-1704. https:// doi.org/10.1007/s00167-020-06031-3

4. Sarac NJ, Sarac BA, Schoenbrunner AR, Janis JE, Harrison RK, Phieffer LS, Quatman CE, Ly TV (2020) A Review of state guidelines for elective orthopaedic procedures during the COVID-19 outbreak. J Bone Joint Surg Am 13. https://doi.org/10.2106/JBJS. 20.00510

5. Mavrogenis AF, Quaile A, Scarlat MM (2020) The virus crisis affects orthopaedic surgery and scientific activities worldwide. Int Orthop 44(5):813-817. https://doi.org/10.1007/s00264-02004557-2

6. Hernigou J, Morel X, Callewier A, Bath O, Hernigou P (2020) Staying home during "COVID-19" decreased fractures, but trauma did not quarantine in one hundred and twelve adults and twenty eight children and the "tsunami of recommendations" could not lockdown twelve elective operations. Int Orthop 25:1-8. https:// doi.org/10.1007/s00264-020-04619-5

7. Hernigou J, Cornil F, Poignard A, El Bouchaibi S, Mani J, Naouri JF, Younes P, Hernigou P (2020) Thoracic computerised tomography scans in one hundred eighteen orthopaedic patients during the COVID-19 pandemic: identification of chest lesions; added values; help in managing patients; burden on the computerised tomography scan department. Int Orthop 7:1-10. https://doi.org/10.1007/ s00264-020-04651-5

8. Petrone B, Fakhoury J, Matai P, Bitterman A, Cohn RM, Lutsky L (2020) Predicting elective orthopaedic sports medicine surgical cancellations based on patient demographics. Arthrosc Sports Med Rehabil 2(2):e83-e89. https://doi.org/10.1016/j.asmr.2019. 11.004

9. Liu D, Zhang W, Pan F, Li L, Yang L, Zheng D, Wang J, Liang B (2020) The pulmonary sequalae in discharged patients with COVID-19: a short-term observational study. Version 2. Respir Res 21(1):125. https://doi.org/10.1186/s12931-020-01385-1

10. Levi M, Thachil J, Iba T, Levy JH (2020) Coagulation abnormalities and thrombosis in patients with COVID-19. Lancet Haematol 7(6):e438-e440. https://doi.org/10.1016/S2352-3026(20)30145-9
11. Romanò CL, Drago L, Del Sel H, Johari A, Lob G, Mavrogenis AF, Benzakour T, World Association against Infection in Orthopedics and Trauma (WAIOT) Study Group on Bone and Joint Infection Definitions (2020) Loud and silent epidemics in the third millennium: tuning-up the volume. Int Orthop. 44(6):1019-1022. https:// doi.org/10.1007/s00264-020-04608-8

12. Wu Z, McGoogan JM (2020) Characteristics of and important lessons from the coronavirus disease 2019 (COVID-19) outbreak in China: summary of a report of 72314 cases from the Chinese center for disease control and prevention. JAMA. 323(13):1239-1242. https://doi.org/10.1001/jama.2020.2648

13. Lezak BA, Cole PA Jr, Schroder LK, Cole PA (2020) Global experience of orthopaedic trauma surgeons facing COVID-19: a survey highlighting the global orthopaedic response. Int Orthop 13:111. https://doi.org/10.1007/s00264-020-04644-4

14. Vaccaro AR, Getz CL, Cohen BE, Cole BJ, Donnally CJ 3rd (2020) Practice management during the COVID-19 pandemic. J Am Acad Orthop Surg 28(11):464-470. https://doi.org/10.5435/JAAOS-D20-00379

15. Luengo-Alonso G, Pérez-Tabernero FG, Tovar-Bazaga M, Arguello-Cuenca JM, Calvo E (2020) Critical adjustments in a department of orthopaedics through the COVID-19 pandemic. Int Orthop 30:1-8. https://doi.org/10.1007/s00264-020-04647-1

16. Zagra L, Faraldi M, Pregliasco F, Vinci A, Lombardi G, Ottaiano I, Accetta R, Perazzo P, D'Apolito R (2020) Changes of clinical activities in an orthopaedic institute in North Italy during the spread of COVID-19 pandemic: a seven-week observational analysis. Int Orthop. 24:1-8. https://doi.org/10.1007/s00264-020-04590-1

17. Grassi A, Pizza N, Tedesco D, Zaffagnini S (2020) The COVID-19 outbreak in Italy: perspectives from an orthopaedic hospital. Int Orthop 22:1-5. https://doi.org/10.1007/s00264-020-04617-7

18. Rubinger L, Gazendam A, Ekhtiari S, Nucci N, Payne A, Johal H, Khanduja V, Bhandari M (2020) Maximizing virtual meetings and conferences: a review of best practices. Int Orthop 23:1-6. https:// doi.org/10.1007/s00264-020-04615-9

19. Vaid S, Kalantar R, Bhandari M (2020) Deep learning COVID-19 detection bias: accuracy through artificial intelligence. Int Orthop. 27:1-4. https://doi.org/10.1007/s00264-020-04609-7

20. Vaid S, McAdie A, Kremer R, Khanduja V, Bhandari M (2020) Risk of a second wave of Covid-19 infections: using artificial intelligence to investigate stringency of physical distancing policies in North America. Int Orthop. https://doi.org/10.1007/s00264-02004653-3

Publisher's note Springer Nature remains neutral with regard to jurisdictional claims in published maps and institutional affiliations. 